\title{
SPECKLE INTERFEROMETRY AT THE OBSERVATORIO ASTRONÓMICO NACIONAL. VII
}

\author{
V. G. Orlov \\ Instituto de Astronomía, Universidad Nacional Autónoma de México, Ciudad de México, México.
}

Received August 25 2020; accepted September 22 2020

\begin{abstract}
The results of speckle interferometric measurements of binary stars performed during June, 2016 with the $2.1 \mathrm{~m}$ telescope at the Observatorio Astronómico Nacional at SPM (México) are given. We report 480 astrometric measurements of 468 double stars systems. The measured angular separations $\rho$ range from $0^{\prime \prime} .091$ to $5^{\prime \prime} .93$. Most of the observed pairs (414 out of 468) are close double stars having separations of $\rho \leq 1^{\prime \prime}$. We confirm as double stars 59 targets and we found 3 new pairs with separation of less than $1^{\prime \prime}$. Finally, we show that the high resolution autocorrelation function in polar coordinates allows to easily recover astrometric parameters even in the presence of strong telescope aberrations.
\end{abstract}

\section{RESUMEN}

Se presentan las mediciones de interferometría de motas de estrellas binarias, realizadas durante el mes de junio de 2016 con el telescopio de $2.1 \mathrm{~m}$ del Observatorio Astronómico Nacional en SPM (México). Reportamos 480 mediciones astrométricas de 468 sistemas de estrellas dobles. Las separaciones angulares medidas $\rho$ van desde $0^{\prime \prime} .091$ a $5^{\prime \prime} .93$. La mayoría de los pares observados (414 de 468) son estrellas dobles cercanas con una separación $\rho \leq 1^{\prime \prime}$. Confirmamos 59 objetos como estrellas dobles e identificamos 3 nuevos pares con una separación de menos de $1^{\prime \prime}$. Finalmente, mostramos que la función de autocorrelación de alta resolución en coordenadas polares permite recuperar fácilmente los parámetros astrométricos, incluso en presencia de fuertes aberraciones del telescopio.

Key Words: binaries: close — techniques:high angular resolution — techniques: interferometric

\section{INTRODUCTION}

In this paper we report astrometric results for double stars obtained by speckle interferometric observations carried out with the $2.1 \mathrm{~m}$ telescope of Sierra San Pedro Mártir National Astronomical Observatory (OAN-SPM) in June of 2016. This is the seventh in a series of publications that started with speckle interferometric measurements performed with the OAN telescopes in 2008 (Orlov et al. 2009). As in our previous publications, we focus on double stars from the Washington Double Star (WDS) catalog (Worley \& Douglass 1997).

The Speckle Interferometry (SI) (Labeyrie 1970) is one of the most used high resolution techniques. This method allows the observer to obtain information about relative positions in close binary stars systems with diffraction-limited accuracy. This tech- nique was most widely used in the study of binary and multiple stars (Tokovinin et al. 2020; Guerrero et al. 2020; Mitrofanova et al. 2020). The observation methodology and data processing of SI is very well studied and described by Tokovinin et al. (2010).

The $2.1 \mathrm{~m}$ telescope of OAN-SPM has a thin primary mirror; its shape is corrected by air bags. The process of correction takes about one hour and is performed only once before observations. During the night, the temperature of the primary mirror and of the telescope mount change, which leads to thermal deformations. Also, corrections introduced by the airbags depend on the hour angle and the zenith distance of the target. As a result, we have different aberrations for each object (Figure 1). Because of this, it is unfeasible to construct a universal synthetic speckle interferometric transfer function or even to 


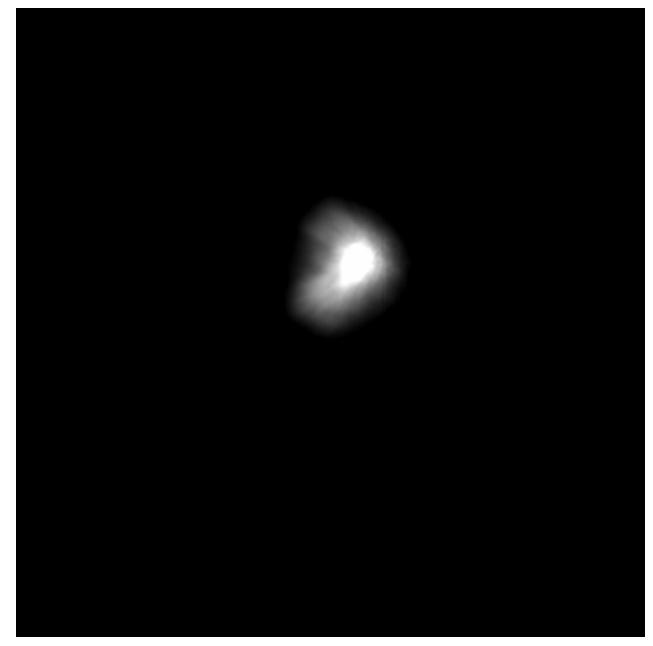

(a)

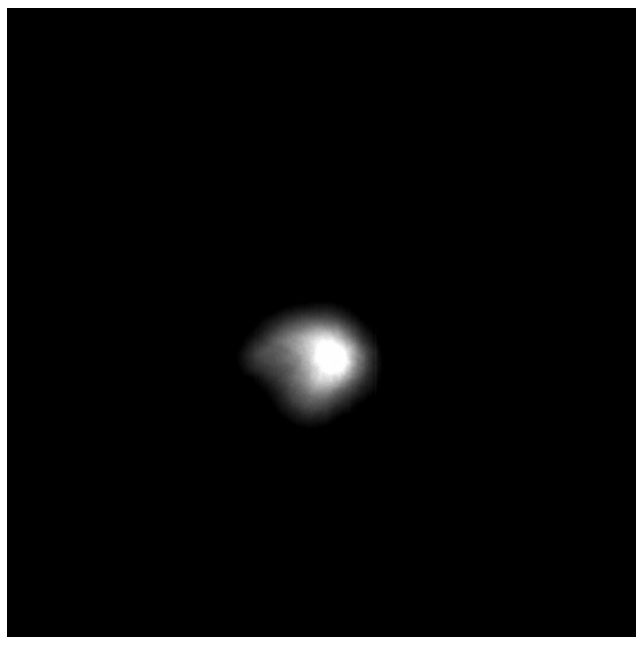

(b)

Fig. 1. Long exposure image of WDS 20480+3917 (a). Long exposure image of WDS 18003+2154 (b). Both images show very strong coma aberration with different angles.

use a reference star. This fact limits the possibility of finding both astrometric and photometric parameters of double stars. In addition, the telescope's vibration distorts the specklegrams. All these factors have a greater impact on the ability to recover photometric parameters than on the recovery of astrometric parameters. Therefore, in this study we focus on improving the recovery of astrometric parameters.

In order to estimate astrometric parameters, we designed an algorithm which allows one to recover each measurement from the distorted power spectrum. In section 3.2 we describe the calculation of the high resolution autocorrelation function in polar coordinates. This algorithm allows for blind searching of the astrometric parameters $\rho$ and $\theta$ of double stars, since it finds the coordinates of the absolute maximum of a two-dimensional discrete function.

\section{OBSERVATIONS}

Speckle interferograms were taken during four nights in the summer of 2016, from June 28 to July 1 at the $2.1 \mathrm{~m}$ telescope of the Observatorio Astronómico Nacional (OAN), which is located at the astronomical site Sierra San Pedro Mártir, México.

The observations were performed using the EMCCD iXon Ultra 888 from Andor Technology. This is a low-noise, high-sensitivity EMCCD camera that can be cooled thermoelectrically down to $-95^{\circ} \mathrm{C}$ which provides excellent elimination of dark noise, even for the short time exposures. The detector has quantum efficiency higher than $80 \%$ in the range of $450-750 \mathrm{~nm}$, with a maximum of $95 \%$ at $550 \mathrm{~nm}$
( $V$-band). This camera allows a fast frame rate so it can be used for speckle interferometry. The detector has $1024 \times 1024$ square pixels of $13 \mu \mathrm{m}$ per side.

The observations were carried out using broadband filters $V(538 / 98 \mathrm{~nm}), R(630 / 118 \mathrm{~nm})$ and $I(894 / 330 \mathrm{~nm})$ from the Johnson-Cousins set. The size of the diffraction-limited speckle $(\lambda / D)$ for the $2.1 \mathrm{~m}$ telescope is approximately 70 mas at this filter wavelength. Given these parameters, we need an angular pixel scale of about 35 mas to obtain a Nyquist sampling of specklegrams. To provide a suitable sampling, we used the $f / 7.5$ secondary mirror combined with a microscope objective lens $\times 4$.

We recorded 500 speckle frames of $400 \times 400$ pixel per object, taken with exposure times of $29.5 \mathrm{~ms}$. We use EM gain of $1 / 300$ photons $/ e^{-}$for all observations.

The seeing was better than $1^{\prime \prime}$ over all the observing nights. However, aberrations introduced by the telescope have a larger effect (Figure 1). As a result, long exposure images have a resolution of about $1.5^{\prime \prime}$.

\section{DATA PROCESSING}

The first step of the data processing is the dark field correction of detected images $I_{n}^{\prime}(\mathbf{x})$ :

$$
I_{n}(\mathbf{x})=I_{n}^{\prime}(\mathbf{x})-\operatorname{Dark}(\mathbf{x}),
$$

where $\mathbf{x}$ is a $2 \mathrm{D}$ spatial coordinate, $I_{n}(\mathbf{x})$ is the corrected image, $\operatorname{Dark}(\mathbf{x})$ is the average dark image captured with a closed shutter (Figure 2 left). In order to remove the reading noise, we also set to zero all values less than $4 \sigma$ of dark (Figure 2 right). 

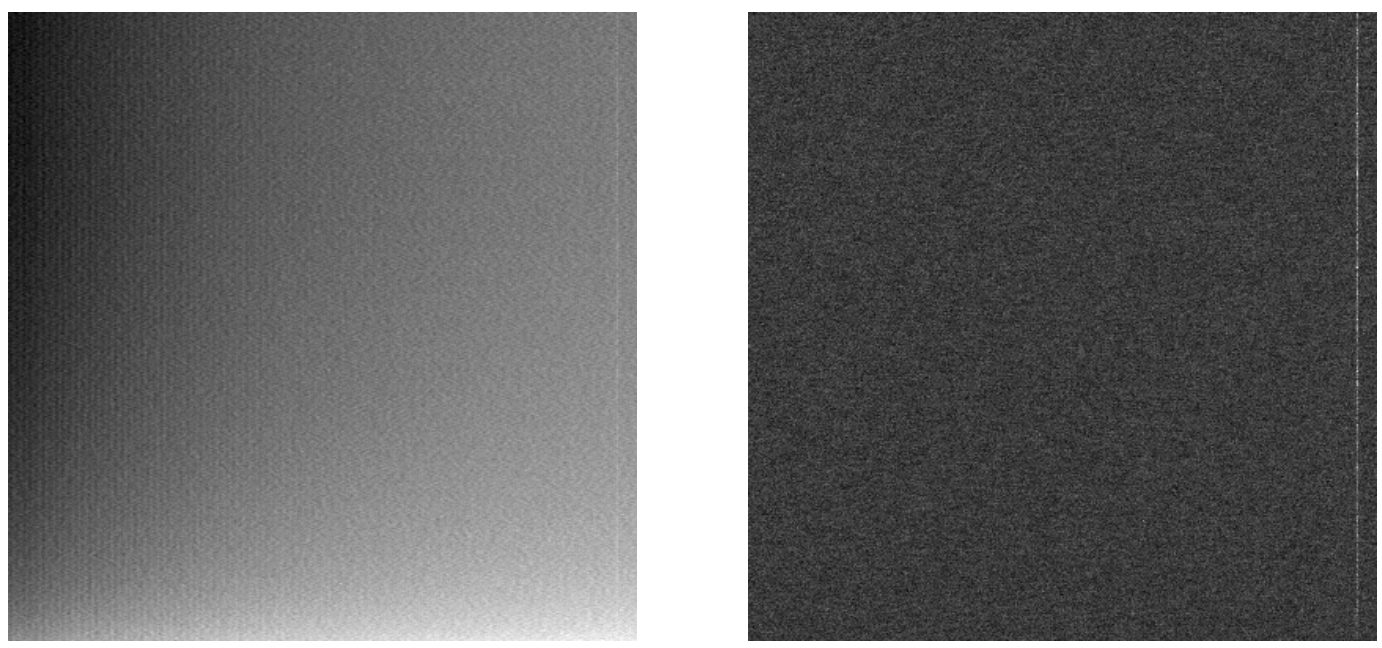

Fig. 2. The average Dark image (left) and $\sigma$ of Dark image (right).
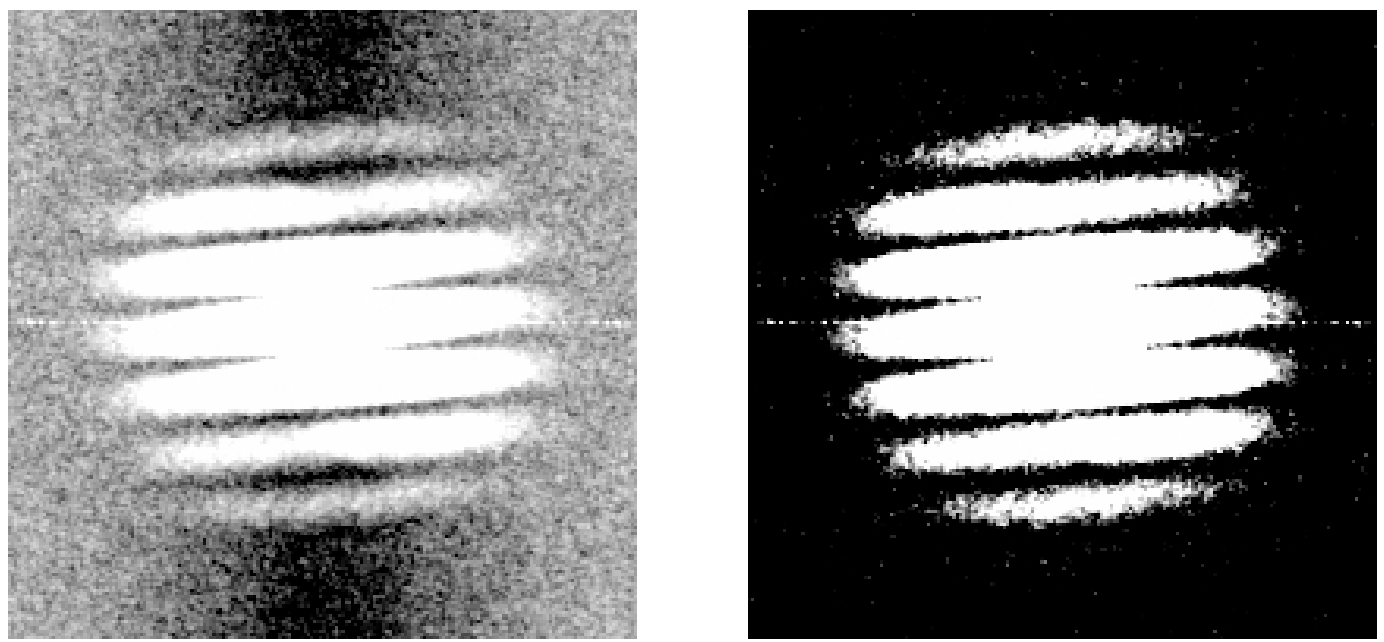

Fig. 3. Power spectrum of WDS $20312+1116$ before photon bias correction (left) and after correction (right). The separation is $0 .{ }^{\prime \prime} 3$.

\subsection{Unshifted Power Spectrum}

The next step is to calculate the averaged power spectrum (PS) for each star:

$$
P S(\mathbf{f})=\left\langle\left|F T\left\{I_{n}(\mathbf{x})\right\}\right|^{2}\right\rangle,
$$

where $\mathbf{f}$ is a spatial frequency, $F T\{\ldots\}$ is the Fourier transform and $\langle\ldots\rangle$ denotes averaging over all images.

In the case of low light images, the averaged power spectrum can be expressed as (Kerp et al. 1992):

$$
P S(\mathbf{f})=P(\mathbf{f}) \cdot|G(\mathbf{f})|^{2}+q|G(\mathbf{f})|^{2},
$$

where $P(\mathbf{f})$ is the unshifted estimation of the power spectrum, $q$ is some constant, $|G(\mathbf{f})|^{2}$ is the power spectrum of the photon event shape function, also known as photon bias. The photon bias $|G(\mathbf{f})|^{2}$ can be determined as the normalized power spectrum of the night sky. $|G(\mathbf{f})|^{2}$ is constant in the $Y$ direction for this camera. Thus, it can be determined directly from $P S(\mathbf{f})$ (Figure 3, left) by analysis of its part beyond the cut-off frequency of telescope. The unshifted power spectrum of specklegrams $P(\mathbf{f})$ is shown in Figure 3 (right). Therefore, it can be presented as:

$$
P(\mathbf{f})=|O(\mathbf{f})|^{2}\left\langle\left|S_{n}(\mathbf{f})\right|^{2}\right\rangle,
$$

where $|O(\mathbf{f})|^{2}$ is the power spectrum of the object, and $\left\langle\left|S_{n}(\mathbf{f})\right|^{2}\right\rangle$ is the speckle interferometric transfer 


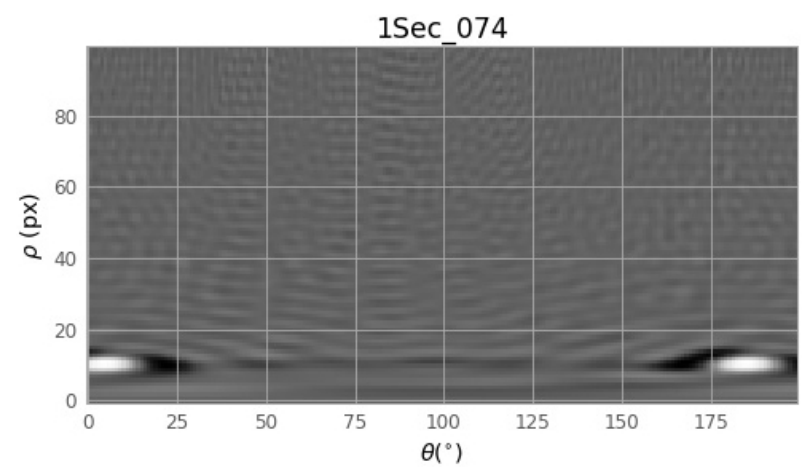

Fig. 4. The ACF in polar coordinates for WDS $20312+1116$. The separation is $0 .{ }^{\prime \prime} 3$.

function. The speckle interferometric transfer function can be obtained by observing a reference star, or one can construct a universal synthetic speckle interferometric transfer function (Tokovinin et al. 2010). If one needs only astrometric parameters, they can be obtained without the speckle interferometric transfer function, directly from $\mathrm{P}(\mathbf{f})$.

\subsection{Autocorrelation Function in Polar Coordinates}

In order to find astrometric parameters from the unshifted power spectrum we calculated the high resolution autocorrelation function in polar coordinates $A C F_{p}$ :

$$
\begin{aligned}
A C F_{p}(\rho, \theta)= & \text { const } \int_{0}^{\infty} \int_{0}^{2 \pi} \cos (2 \pi r \rho \cos (\theta-\phi)) \times \\
& \times P(r, \phi) W(r, \phi) r d r d \phi
\end{aligned}
$$

where $W(r, \phi)$ is the window which excludes part of $P(r, \phi)$ beyond the cut-off frequency of the telescope $f_{T}$ and for frequencies lower than the atmospheric cutoff $f_{A}$. Also, taking in to account central symmetry of $P(r, \phi)$ equation 5 can be rewritten as:

$$
\begin{aligned}
\operatorname{ACF}_{p}(\rho, \theta)= & \text { const } \int_{f_{A}}^{f_{T}} \int_{0}^{\pi} \cos (2 \pi r \rho \cos (\theta-\phi)) \times \\
& \times P(r, \phi) r d r d \phi .
\end{aligned}
$$

One example of $A C F_{p}$ is shown in Figure 4. The position of the maximum gives us $\rho$ and $\theta$ which determine the position of the component in the coordinates of the detector.

Now let us see if $A C F_{p}$ allows us to find astrometric parameters when the power spectrum is distorted by vibrations and strong aberrations of the telescope. As shown in Figure 5 (left) the power spectrum loses the high frequencies in the vertical direction. However, the high resolution $A C F_{p}$ has a strong maximum (Figure 5, right). The precision of determining astrometric parameters of the binary system depends on the accuracy with which we can determine the coordinates of the maximum of the discrete function $A C F_{p}$. Thus, we can recover the astrometric parameters from the distorted power spectrum. Although measurements can be carried out without the speckle interferometric transfer function correction, its use improves their accuracy.

\subsection{Degree Ambiguity}

The power spectrum has a $180^{\circ}$ ambiguity. To deal with this issue, we used the self-calibrating shift-andadd technique (Christou et al. 1986). The technique allows us to get diffraction-limited images without using any reference star. When the components have similar magnitudes, the result of this technique is similar to the diffraction-limited autocorrelation, as in Figure 6 (left), contrary to the case in which there is a clear difference between the components, as shown in Figure 6 (right). This double star has a difference of one magnitude between components. This technique allows us to overcome the common 180 degree ambiguity, thus obtaining a reconstruction of the close double star system. Then we can obtain the real $\theta$ (position angle) and $\rho$ (separation) by calibration.

\subsection{Calibration}

To perform the calibration, we need to find the pixel scale and the position angle offset. There are two common ways to do this. The first one is by observing some binary stars which have known orbits of grade 1 and calculating ephemerides from the orbital elements. The second way is by observing double stars with very slow relative motion of the components. In this case, ephemerides are calculated by linear approximation of the component motion, or by using the last known value of $\rho$ and $\theta$ if there is no evidence of motion over more than 20 years. Most suitable for this method are optical doubles with slow proper motion. This method is preferable to the first one, because the accuracy of speckle interferometric measurements with 2-meter telescopes exceeds the accuracy of even the best orbits (Tokovinin et al. 2015).

For the astrometric calibration, we selected 21 systems with a separation ranging from $4^{\prime \prime}$ to $6^{\prime \prime}$ which had more than one reliable observation from the Fourth Catalog of Interferometric Measurements 

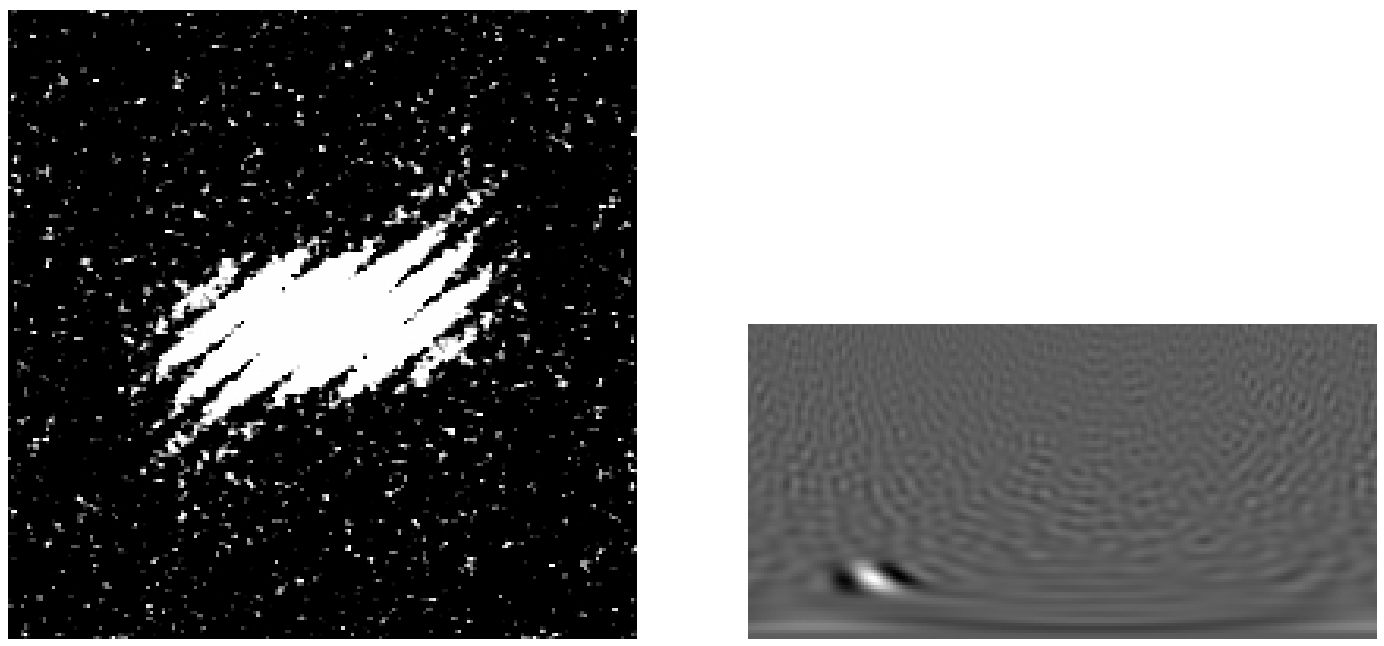

Fig. 5. Power spectrum $P(\mathbf{f})$ of WDS 14394-0733 distorted by vibrations and aberrations of the telescope (left). $A C F_{p}$ of WDS 14394-0733 obtained from its $P(\mathbf{f})$ (right). The separation is $0 .^{\prime \prime} 55$.
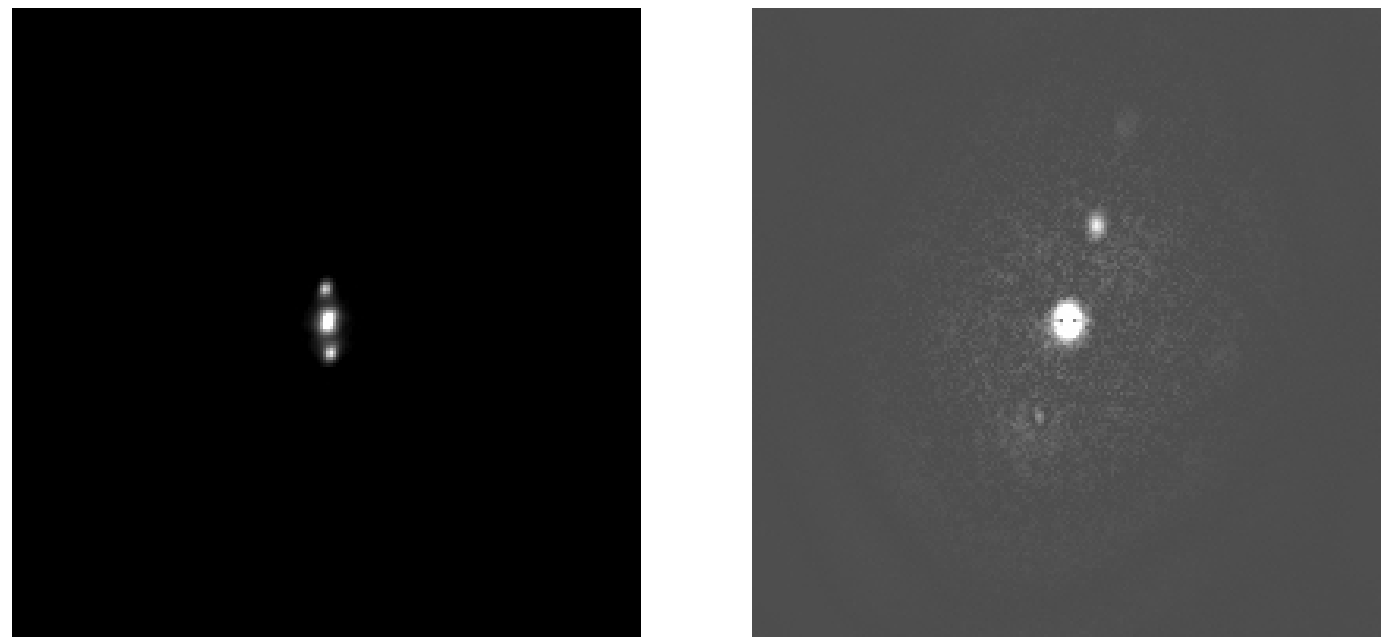

Fig. 6. Example of the reconstruction of WDS 20312+1116 (left) and WDS $19326+0435, \Delta m=1$ (right).

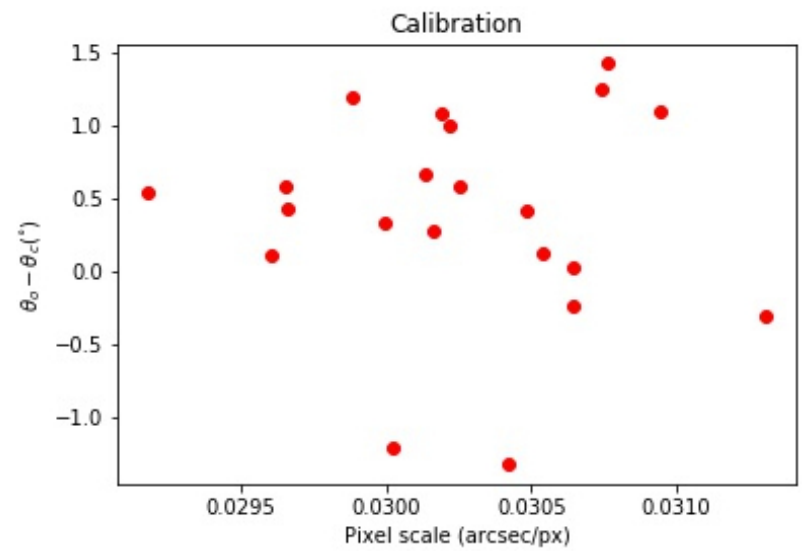

of Binary Stars (Hartkopf et al. 2001) and from the WDS catalog. These 21 systems also have very slow movements and a long time base of observations. A comparison with our data (Figure 7) gives us the following offset for the position angle $\theta_{0}=-0.42^{\circ} \pm$ $0.14^{\circ}$ and a pixel scale $s=0^{\prime \prime} .03026 \pm 0^{\prime \prime} .00009$ per pixel.

\section{ASTROMETRIC MEASUREMENTS}

The astrometric measurements we obtained for double stars are displayed in four tables (Tables 1-4). Table 1 presents astrometric measurements of 21 double stars used for calibration. All these systems show slow motions of components. The first

Fig. 7. Calibration. column contains the epoch-2000 coordinates in the 
TABLE 1

WIDE DOUBLE STARS WITH VERY SLOW RELATIVE MOTION

\begin{tabular}{|c|c|c|c|c|c|}
\hline $\begin{array}{l}\text { WDS } \\
(2000)\end{array}$ & $\begin{array}{c}\text { Discoverer } \\
\text { designation }\end{array}$ & $\begin{array}{c}\text { Epoch } \\
\text { Julian year }\end{array}$ & Fil. & $\begin{array}{c}\theta \\
\left({ }^{\circ}\right)\end{array}$ & $\begin{array}{c}\rho \\
\left({ }^{\prime \prime}\right)\end{array}$ \\
\hline $14083+2112$ & STF1804 & 2016.4882 & I & $14.01 \pm 0.14$ & $4.796 \pm 0.014$ \\
\hline $14100+0401$ & STF 1805 & 2016.4882 & I & $33.60 \pm 0.14$ & $4.837 \pm 0.014$ \\
\hline $14134+0524$ & STF1813 & 2016.4882 & I & $193.54 \pm 0.14$ & $4.703 \pm 0.014$ \\
\hline $14165+2007$ & STF 1825 & 2016.4882 & I & $153.51 \pm 0.14$ & $4.369 \pm 0.013$ \\
\hline $14279+2123$ & HO 543 & 2016.4882 & I & $237.37 \pm 0.14$ & $4.631 \pm 0.014$ \\
\hline $14506-0001$ & STF1885 & 2016.4882 & I & $145.70 \pm 0.14$ & $4.100 \pm 0.012$ \\
\hline $15589+2147$ & STF1990 BC & 2016.4883 & I & $26.34 \pm 0.14$ & $4.048 \pm 0.012$ \\
\hline $16003+1140$ & STF1992 AB,C & 2016.4883 & I & $326.35 \pm 0.14$ & $5.946 \pm 0.018$ \\
\hline $17178+0733$ & $\mathrm{~J} 450$ & 2016.4896 & $\mathrm{R}$ & $60.99 \pm 0.14$ & $4.651 \pm 0.014$ \\
\hline $17268+2240$ & J 1032 & 2016.4896 & $\mathrm{R}$ & $350.78 \pm 0.14$ & $4.057 \pm 0.012$ \\
\hline $17324+2352$ & STF2182 AB & 2016.4896 & $\mathrm{R}$ & $0.86 \pm 0.14$ & $5.418 \pm 0.016$ \\
\hline $17362+0637$ & STF2188 & 2016.4896 & $\mathrm{R}$ & $203.71 \pm 0.14$ & $5.516 \pm 0.016$ \\
\hline $17590+0202$ & STF2252 AB & 2016.4896 & $\mathrm{R}$ & $24.26 \pm 0.14$ & $3.948 \pm 0.012$ \\
\hline $18106+0349$ & FOX 220 & 2016.4896 & $\mathrm{R}$ & $75.37 \pm 0.14$ & $5.977 \pm 0.018$ \\
\hline $18154+1946$ & STT 346 & 2016.4896 & $\mathrm{R}$ & $329.58 \pm 0.14$ & $5.208 \pm 0.015$ \\
\hline $18206+2248$ & STF2310 & 2016.4896 & $\mathrm{R}$ & $237.96 \pm 0.14$ & $5.052 \pm 0.015$ \\
\hline $18247-0636$ & STF2313 & 2016.4896 & $\mathrm{R}$ & $196.00 \pm 0.14$ & $5.857 \pm 0.017$ \\
\hline $18258+0359$ & J 462 & 2016.4896 & $\mathrm{R}$ & $348.32 \pm 0.14$ & $4.180 \pm 0.012$ \\
\hline $18266+0627$ & GCB 31 & 2016.4896 & $\mathrm{R}$ & $75.87 \pm 0.14$ & $4.501 \pm 0.013$ \\
\hline
\end{tabular}

TABLE 2

ASTROMETRIC MEASUREMENTS OF THE OBSERVED DOUBLE STARS WITH NO CALCULATED ORBITS

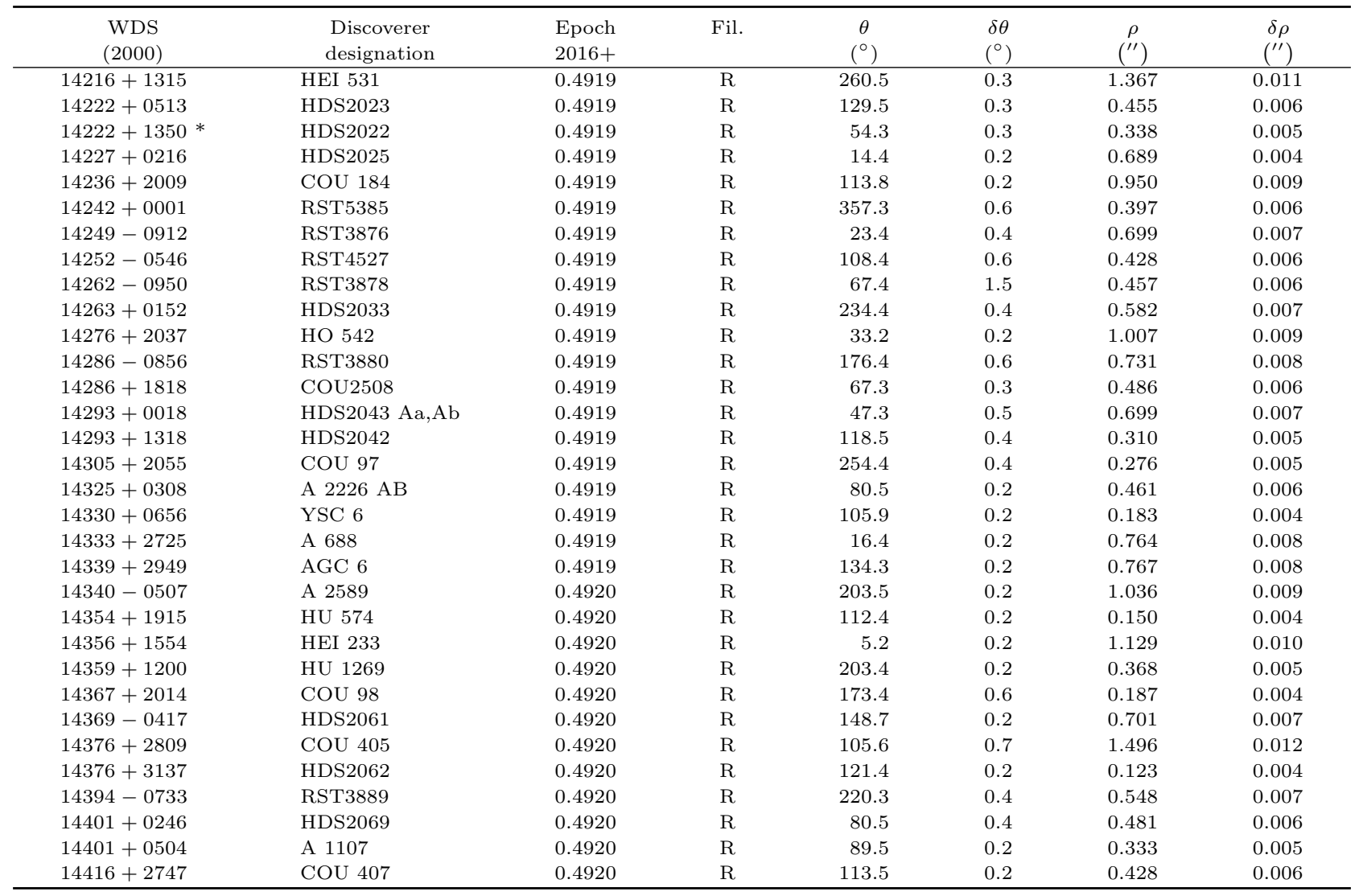


TABLE 2. CONTINUED

\begin{tabular}{|c|c|c|c|c|c|c|c|}
\hline $14417+0932$ & STF1866 & 0.4920 & $\mathrm{R}$ & 205.2 & 0.2 & 0.761 & 0.008 \\
\hline $14419+1847$ & COU 185 & 0.4920 & $\mathrm{R}$ & 305.9 & 0.3 & 0.899 & 0.009 \\
\hline $15261+1810$ & STF1940 & 0.4974 & $\mathrm{R}$ & 332.4 & 0.2 & 0.370 & 0.005 \\
\hline $15262+1418$ & HEI 236 & 0.4974 & $\mathrm{R}$ & 107.5 & 0.3 & 0.523 & 0.006 \\
\hline $15268+2840$ & $\mathrm{COU} 484$ & 0.4975 & $\mathrm{R}$ & 263.4 & 0.2 & 0.333 & 0.005 \\
\hline $16013-0658$ & BU 623 & 0.4920 & $\mathrm{R}$ & 225.6 & 0.2 & 0.733 & 0.008 \\
\hline $16043-0313$ & RST4558 & 0.4921 & $\mathrm{R}$ & 170.3 & 0.3 & 0.580 & 0.007 \\
\hline $16049+0213$ & HEI 793 & 0.4921 & $\mathrm{R}$ & 196.1 & 0.2 & 1.523 & 0.012 \\
\hline $16062+1809$ & COU2389 & 0.4921 & $\mathrm{R}$ & 238.5 & 0.2 & 1.126 & 0.010 \\
\hline $16071+1654$ & BU 812 & 0.4921 & $\mathrm{R}$ & 97.4 & 0.2 & 0.731 & 0.007 \\
\hline $16072+1848 *$ & COU 196 & 0.4921 & $\mathrm{R}$ & 349.6 & 1.9 & 1.584 & 0.013 \\
\hline $16080+0559$ & TDS9770 & 0.4921 & $\mathrm{R}$ & 256.3 & 0.3 & 0.582 & 0.007 \\
\hline $16087+0524$ & HDS2278 & 0.4921 & $\mathrm{R}$ & 112.4 & 0.2 & 0.281 & 0.005 \\
\hline $16092-0549$ & RST4559 & 0.4921 & $\mathrm{R}$ & 296.4 & 0.4 & 0.975 & 0.009 \\
\hline $16092-1057^{*}$ & HDS2279 & 0.4921 & $\mathrm{R}$ & 325.5 & 0.4 & 0.335 & 0.005 \\
\hline $16097-0633$ & RST3932 & 0.4921 & $\mathrm{R}$ & 137.4 & 0.8 & 0.212 & 0.005 \\
\hline $16139+0123$ & RST5407 & 0.4921 & $\mathrm{R}$ & 223.3 & 0.2 & 0.972 & 0.009 \\
\hline $16152-0709$ & RST3938 & 0.4921 & $\mathrm{R}$ & 209.5 & 0.3 & 1.249 & 0.011 \\
\hline $16168+1447$ & HDS2301 & 0.4921 & $\mathrm{R}$ & 55.4 & 0.2 & 1.007 & 0.009 \\
\hline $16169+1948$ & COU 107 & 0.4921 & $\mathrm{R}$ & 114.4 & 0.2 & 0.637 & 0.007 \\
\hline $16173+1626$ & YSC 153 & 0.4921 & $\mathrm{R}$ & 131.4 & 0.3 & 0.338 & 0.005 \\
\hline $16174+0643 *$ & TDS9822 & 0.4921 & $\mathrm{R}$ & 349.6 & 0.4 & 0.546 & 0.007 \\
\hline $16177+1342$ & YSC 154 & 0.4921 & $\mathrm{R}$ & 44.9 & 0.4 & 0.682 & 0.007 \\
\hline $16186+1247$ & HEI 241 & 0.4921 & $\mathrm{R}$ & 59.5 & 0.3 & 0.763 & 0.008 \\
\hline $16581+0902$ & HDS2401 & 0.4922 & $\mathrm{R}$ & 17.5 & 0.2 & 0.332 & 0.005 \\
\hline $16581+1509$ & STT 319 & 0.4922 & $\mathrm{R}$ & 65.8 & 0.1 & 0.858 & 0.008 \\
\hline $16584+1358$ & YSC 61 & 0.4922 & $\mathrm{R}$ & 260.4 & 1.0 & 0.608 & 0.007 \\
\hline $16594+1419$ & STT 321 & 0.4922 & $\mathrm{R}$ & 15.3 & 0.2 & 0.581 & 0.007 \\
\hline $16595+0942$ & $\mathrm{BU} 1298 \mathrm{AB}$ & 0.4922 & $\mathrm{R}$ & 133.3 & 0.2 & 0.429 & 0.006 \\
\hline $17003+0106$ & A 2235 & 0.4922 & $\mathrm{R}$ & 269.4 & 0.3 & 0.827 & 0.008 \\
\hline $17012+0627 *$ & HDS2409 & 0.4922 & $\mathrm{R}$ & 37.4 & 0.7 & 0.274 & 0.005 \\
\hline $17042+1834 *$ & TDT 197 & 0.4922 & $\mathrm{R}$ & 91.4 & 0.4 & 0.396 & 0.006 \\
\hline $17046-0339$ & RST4565 & 0.4922 & $\mathrm{R}$ & 164.6 & 0.3 & 0.823 & 0.008 \\
\hline $17050+0724$ & TDT 204 & 0.4922 & $\mathrm{R}$ & 152.3 & 0.3 & 0.638 & 0.007 \\
\hline $17080-0957$ & RST3966 & 0.4922 & $\mathrm{R}$ & 111.0 & 1.2 & 0.487 & 0.006 \\
\hline $17086+0951$ & HU 167 & 0.4922 & $\mathrm{R}$ & 272.6 & 0.5 & 0.820 & 0.008 \\
\hline $17088+0002$ & A 2237 & 0.4922 & $\mathrm{R}$ & 69.9 & 0.3 & 0.918 & 0.009 \\
\hline $17107+1651$ & HEI 167 & 0.4922 & $\mathrm{R}$ & 98.4 & 0.3 & 0.394 & 0.006 \\
\hline $17107+2104 *$ & TDT 251 & 0.4922 & $\mathrm{R}$ & 122.4 & 0.4 & 0.544 & 0.007 \\
\hline $17107+2312 *$ & TDT 250 & 0.4922 & $\mathrm{R}$ & 11.0 & 0.3 & 0.795 & 0.008 \\
\hline $17110+0302$ & HDS2426 & 0.4922 & $\mathrm{R}$ & 195.4 & 0.3 & 0.883 & 0.009 \\
\hline $17110+1622$ & HEI 168 & 0.4922 & $\mathrm{R}$ & 65.7 & 0.2 & 0.763 & 0.008 \\
\hline $17136+0405$ & HEI 895 & 0.4922 & $\mathrm{R}$ & 15.5 & 1.7 & 0.854 & 0.009 \\
\hline $17140+2119$ & COU 111 & 0.4923 & $\mathrm{R}$ & 249.5 & 0.3 & 0.613 & 0.007 \\
\hline $17142+2731$ & COU 495 & 0.4923 & $\mathrm{R}$ & 100.6 & 0.4 & 0.819 & 0.008 \\
\hline $17150+1238 *$ & HDS2439 & 0.4923 & $\mathrm{R}$ & 168.9 & 0.5 & 0.518 & 0.006 \\
\hline $17155+2007$ & HU 489 & 0.4923 & $\mathrm{R}$ & 35.8 & 0.2 & 1.035 & 0.009 \\
\hline $17160+1702 *$ & TDT 294 & 0.4923 & $\mathrm{R}$ & 77.6 & 0.5 & 0.582 & 0.007 \\
\hline $17174+1939$ & $\mathrm{COU} 496 \mathrm{AB}$ & 0.4923 & $\mathrm{R}$ & 172.0 & 0.9 & 0.823 & 0.008 \\
\hline $17182+1559$ & HEI 246 & 0.4923 & $\mathrm{R}$ & 45.3 & 0.3 & 1.160 & 0.010 \\
\hline $17247+3802$ & COU1142 AB & 0.4977 & $\mathrm{R}$ & 222.4 & 0.2 & 1.858 & 0.014 \\
\hline $17272+3235 *$ & TDT 362 & 0.4977 & $\mathrm{R}$ & 156.4 & 0.4 & 0.310 & 0.005 \\
\hline $17285+3657$ & COU1143 & 0.4977 & $\mathrm{R}$ & 244.4 & 0.3 & 0.370 & 0.005 \\
\hline $17290+3845$ & COU1297 & 0.4977 & $\mathrm{R}$ & 99.4 & 0.3 & 0.276 & 0.005 \\
\hline $17293+3758$ & HO 417 & 0.4977 & $\mathrm{R}$ & 306.4 & 0.2 & 0.310 & 0.005 \\
\hline $17345+3935$ & COU1298 & 0.4977 & $\mathrm{R}$ & 251.5 & 0.2 & 0.302 & 0.005 \\
\hline $17354+3443$ & COU 995 & 0.4977 & $\mathrm{R}$ & 335.3 & 0.2 & 0.421 & 0.006 \\
\hline $17359+3205$ & COU 807 & 0.4977 & $\mathrm{R}$ & 143.4 & 0.3 & 0.670 & 0.007 \\
\hline $17455+3554$ & HDS2509 & 0.4977 & $\mathrm{R}$ & 74.9 & 0.2 & 0.548 & 0.006 \\
\hline $17462+3853$ & COU1300 & 0.4977 & $\mathrm{R}$ & 125.7 & 0.2 & 0.765 & 0.008 \\
\hline $17464+3553 *$ & TDT 497 & 0.4977 & $\mathrm{R}$ & 263.8 & 0.3 & 0.761 & 0.008 \\
\hline $17470+3750$ & COU1144 & 0.4977 & $\mathrm{R}$ & 283.9 & 0.2 & 0.941 & 0.009 \\
\hline $17471+3235$ & COU 634 & 0.4977 & $\mathrm{R}$ & 79.4 & 0.3 & 0.246 & 0.005 \\
\hline $17504+3526$ & ORL $1 \mathrm{Aa}, \mathrm{Ab}$ & 0.4977 & $\mathrm{R}$ & 29.4 & 0.2 & 0.304 & 0.005 \\
\hline $17512+3821$ & HU 1183 & 0.4977 & $\mathrm{R}$ & 191.4 & 0.2 & 0.489 & 0.006 \\
\hline
\end{tabular}


TABLE 2. (CONTINUED)

\begin{tabular}{|c|c|c|c|c|c|c|}
\hline TDT 553 & 0.4977 & $\mathrm{R}$ & 62.4 & 0.3 & 0.702 & 0.007 \\
\hline A $2987 \mathrm{AC}$ & 0.4977 & $\mathrm{R}$ & 60.9 & 0.2 & 1.402 & 0.012 \\
\hline HU 1184 & 0.4977 & $\mathrm{R}$ & 204.6 & 0.2 & 0.916 & 0.009 \\
\hline $\mathrm{HO} 74 \mathrm{AB}$ & 0.4977 & $\mathrm{R}$ & 124.8 & 0.2 & 3.287 & 0.023 \\
\hline COU1001 Aa,Ab & 0.4977 & $\mathrm{R}$ & 220.5 & 0.2 & 0.483 & 0.006 \\
\hline TDS 893 & 0.4923 & $\mathrm{R}$ & 279.8 & 0.5 & 0.766 & 0.008 \\
\hline COU1000 & 0.4977 & $\mathrm{R}$ & 154.4 & 0.2 & 0.944 & 0.009 \\
\hline COU1002 & 0.4977 & $\mathrm{R}$ & 163.7 & 0.2 & 0.853 & 0.008 \\
\hline COU1458 & 0.4977 & $\mathrm{R}$ & 78.4 & 0.4 & 0.364 & 0.005 \\
\hline COU 115 & 0.4950 & $\mathrm{R}$ & 118.5 & 0.2 & 0.278 & 0.005 \\
\hline $\mathrm{COU} 810$ & 0.4950 & $\mathrm{R}$ & 99.4 & 0.4 & 0.157 & 0.004 \\
\hline TDT 636 & 0.4950 & $\mathrm{R}$ & 185.4 & 0.7 & 0.167 & 0.004 \\
\hline COU1147 & 0.4950 & $\mathrm{R}$ & 178.2 & 0.2 & 0.737 & 0.008 \\
\hline COU1147 & 0.4978 & $\mathrm{R}$ & 178.2 & 0.2 & 0.737 & 0.008 \\
\hline COU2115 & 0.4950 & $\mathrm{R}$ & 41.9 & 0.8 & 0.274 & 0.005 \\
\hline HDS2546 & 0.4950 & $\mathrm{R}$ & 235.5 & 0.4 & 0.578 & 0.007 \\
\hline COU1787 & 0.4951 & $\mathrm{R}$ & 325.4 & 0.3 & 0.395 & 0.006 \\
\hline COU2513 & 0.4951 & $\mathrm{R}$ & 57.6 & 0.2 & 0.915 & 0.009 \\
\hline TDT 678 & 0.4978 & $\mathrm{R}$ & 236.4 & 0.4 & 0.669 & 0.007 \\
\hline HO 79 & 0.4978 & $\mathrm{R}$ & 61.4 & 0.9 & 0.216 & 0.005 \\
\hline TDT 689 & 0.4978 & $\mathrm{R}$ & 242.4 & 1.0 & 0.366 & 0.006 \\
\hline COU2392 & 0.4951 & $\mathrm{R}$ & 147.3 & 0.3 & 0.641 & 0.007 \\
\hline COU1005 AB,C & 0.4978 & $\mathrm{R}$ & 25.4 & 0.4 & 1.889 & 0.015 \\
\hline COU1005 AB & 0.4978 & $\mathrm{R}$ & 333.4 & 0.4 & 0.211 & 0.005 \\
\hline HDS2564 & 0.4951 & $\mathrm{R}$ & 306.4 & 0.3 & 0.364 & 0.005 \\
\hline HDS2565 & 0.4978 & $\mathrm{R}$ & 340.1 & 0.2 & 0.670 & 0.007 \\
\hline A 238 & 0.4924 & $\mathrm{R}$ & 71.6 & 0.2 & 0.636 & 0.007 \\
\hline $\mathrm{HO} 82 \mathrm{AB}, \mathrm{C}$ & 0.4978 & $\mathrm{R}$ & 220.7 & 0.2 & 0.701 & 0.007 \\
\hline COU2117 & 0.4951 & $\mathrm{R}$ & 298.5 & 0.3 & 0.426 & 0.006 \\
\hline STF2292 & 0.4924 & $\mathrm{R}$ & 276.9 & 0.2 & 0.885 & 0.008 \\
\hline MLR 585 & 0.4951 & $\mathrm{R}$ & 335.4 & 0.3 & 0.428 & 0.006 \\
\hline HDS2571 & 0.4951 & $\mathrm{R}$ & 310.4 & 0.4 & 0.274 & 0.005 \\
\hline HDS2573 & 0.4924 & $\mathrm{R}$ & 165.4 & 0.2 & 0.853 & 0.008 \\
\hline TDT 743 & 0.4924 & $\mathrm{R}$ & 67.8 & 1.0 & 1.004 & 0.010 \\
\hline A 1376 & 0.4951 & $\mathrm{R}$ & 203.4 & 0.3 & 0.338 & 0.005 \\
\hline HEI 170 & 0.4924 & $\mathrm{R}$ & 313.4 & 0.4 & 0.337 & 0.005 \\
\hline TDT 749 & 0.4978 & $\mathrm{R}$ & 15.4 & 0.7 & 0.167 & 0.004 \\
\hline HDS2576 & 0.4924 & $\mathrm{R}$ & 68.1 & 0.3 & 0.966 & 0.009 \\
\hline HU 927 & 0.4978 & $\mathrm{R}$ & 102.4 & 0.2 & 0.369 & 0.005 \\
\hline COU1007 & 0.4978 & $\mathrm{R}$ & 38.4 & 0.3 & 0.274 & 0.005 \\
\hline TDT 778 & 0.4978 & $\mathrm{R}$ & 177.4 & 0.5 & 0.914 & 0.009 \\
\hline MLR 586 & 0.4951 & $\mathrm{R}$ & 194.4 & 0.2 & 0.636 & 0.007 \\
\hline COU1306 & 0.4978 & $\mathrm{R}$ & 42.4 & 0.3 & 0.454 & 0.006 \\
\hline COU1460 & 0.4978 & $\mathrm{R}$ & 341.5 & 0.2 & 0.452 & 0.006 \\
\hline MLR 536 & 0.4951 & $\mathrm{R}$ & 194.7 & 0.3 & 0.851 & 0.008 \\
\hline TDT 818 & 0.4978 & $\mathrm{R}$ & 205.4 & 0.4 & 0.273 & 0.005 \\
\hline YSC 66 & 0.4951 & $\mathrm{R}$ & 42.7 & 0.2 & 0.914 & 0.009 \\
\hline HDS2603 & 0.4978 & $\mathrm{R}$ & 353.7 & 0.3 & 0.853 & 0.008 \\
\hline RST4587 & 0.4895 & $\mathrm{R}$ & 332.3 & 0.3 & 0.395 & 0.006 \\
\hline MLR 537 & 0.4951 & $\mathrm{R}$ & 56.5 & 0.2 & 0.579 & 0.007 \\
\hline $\mathrm{HDS} 2604 \mathrm{Aa}, \mathrm{Ab}$ & 0.4924 & $\mathrm{R}$ & 212.1 & 0.3 & 0.729 & 0.008 \\
\hline COU1461 & 0.4978 & $\mathrm{R}$ & 245.1 & 0.3 & 0.790 & 0.008 \\
\hline HDS2607 & 0.4896 & $\mathrm{R}$ & 243.4 & 0.5 & 0.151 & 0.004 \\
\hline STF2316 AB & 0.4896 & $\mathrm{R}$ & 321.7 & 0.2 & 3.749 & 0.026 \\
\hline HDS2614 & 0.4896 & $\mathrm{R}$ & 333.3 & 0.3 & 0.704 & 0.007 \\
\hline TDT 897 & 0.4896 & $\mathrm{R}$ & 169.4 & 0.4 & 0.489 & 0.006 \\
\hline COU 203 & 0.4896 & $\mathrm{R}$ & 60.5 & 0.3 & 0.488 & 0.006 \\
\hline COU 203 & 0.4924 & $\mathrm{R}$ & 60.5 & 1.1 & 0.486 & 0.006 \\
\hline COU 507 & 0.4896 & $\mathrm{R}$ & 154.0 & 0.4 & 0.947 & 0.009 \\
\hline A $581 \mathrm{AB}$ & 0.4896 & $\mathrm{R}$ & 139.5 & 0.3 & 0.392 & 0.006 \\
\hline TDT 910 & 0.4978 & $\mathrm{R}$ & 195.6 & 0.2 & 0.821 & 0.008 \\
\hline TDT 911 & 0.4951 & $\mathrm{R}$ & 196.4 & 0.3 & 0.734 & 0.008 \\
\hline MLR 357 & 0.4951 & $\mathrm{R}$ & 204.3 & 0.4 & 0.582 & 0.007 \\
\hline COU 508 & 0.4896 & $\mathrm{R}$ & 253.7 & 0.3 & 0.910 & 0.009 \\
\hline HU 583 & 0.4896 & $\mathrm{R}$ & 307.3 & 0.2 & 0.759 & 0.008 \\
\hline
\end{tabular}


TABLE 2. (CONTINUED)

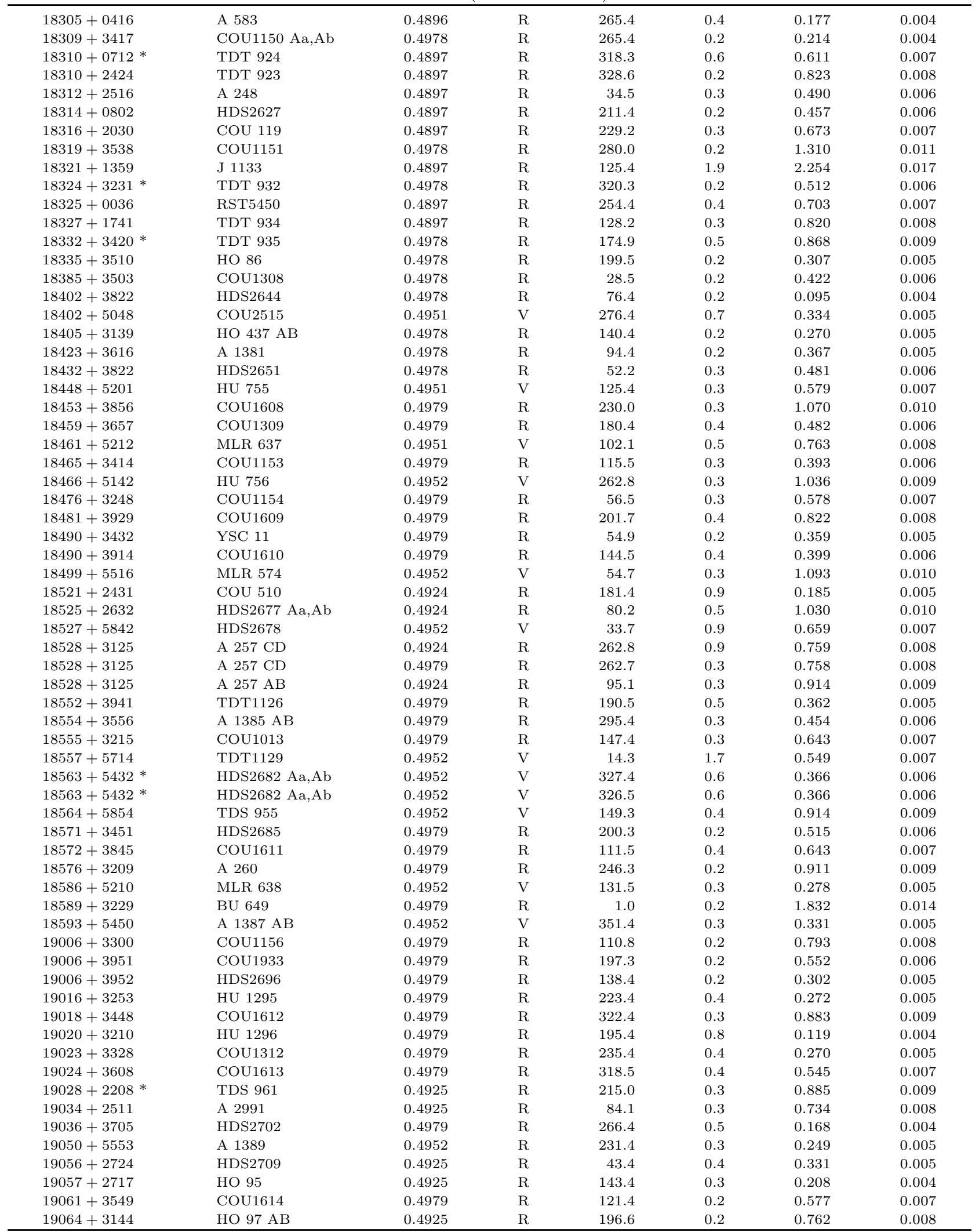


TABLE 2. (CONTINUED)

\begin{tabular}{|c|c|c|c|c|c|}
\hline 0.4925 & $\mathrm{R}$ & 336.3 & 0.2 & 1.063 & 0.010 \\
\hline 0.4979 & $\mathrm{R}$ & 96.4 & 0.4 & 0.426 & 0.006 \\
\hline 0.4925 & $\mathrm{R}$ & 167.4 & 0.3 & 0.396 & 0.006 \\
\hline 0.4979 & $\mathrm{R}$ & 124.3 & 0.4 & 0.550 & 0.007 \\
\hline 0.4952 & $\mathrm{R}$ & 37.4 & 0.3 & 0.392 & 0.006 \\
\hline 0.4952 & $\mathrm{R}$ & 153.3 & 0.3 & 0.973 & 0.009 \\
\hline 0.4952 & $\mathrm{R}$ & 277.2 & 0.3 & 0.762 & 0.008 \\
\hline 0.4925 & $\mathrm{R}$ & 100.7 & 0.3 & 1.035 & 0.009 \\
\hline 0.4952 & $\mathrm{R}$ & 65.6 & 0.3 & 0.855 & 0.008 \\
\hline 0.4952 & $\mathrm{R}$ & 277.4 & 0.2 & 0.151 & 0.004 \\
\hline 0.4952 & $\mathrm{R}$ & 22.4 & 0.6 & 0.186 & 0.004 \\
\hline 0.4952 & $\mathrm{R}$ & 161.4 & 0.3 & 0.093 & 0.004 \\
\hline 0.4952 & $\mathrm{R}$ & 256.4 & 0.2 & 0.704 & 0.007 \\
\hline 0.4952 & $\mathrm{R}$ & 192.4 & 0.3 & 0.248 & 0.005 \\
\hline 0.4952 & $\mathrm{R}$ & 300.3 & 0.3 & 0.819 & 0.008 \\
\hline 0.4952 & $\mathrm{R}$ & 168.7 & 0.2 & 0.857 & 0.008 \\
\hline 0.4952 & $\mathrm{R}$ & 125.4 & 0.3 & 0.515 & 0.006 \\
\hline 0.4952 & $\mathrm{R}$ & 170.6 & 0.2 & 1.007 & 0.009 \\
\hline 0.4925 & $\mathrm{R}$ & 6.4 & 0.3 & 0.271 & 0.005 \\
\hline 0.4925 & $\mathrm{R}$ & 208.9 & 0.3 & 0.792 & 0.008 \\
\hline 0.4897 & $\mathrm{R}$ & 253.4 & 0.3 & 0.184 & 0.004 \\
\hline 0.4897 & $\mathrm{R}$ & 290.4 & 1.0 & 0.362 & 0.006 \\
\hline 0.4897 & $\mathrm{R}$ & 16.8 & 0.5 & 0.729 & 0.008 \\
\hline 0.4897 & $\mathrm{R}$ & 226.7 & 0.4 & 0.885 & 0.009 \\
\hline 0.4897 & $\mathrm{R}$ & 324.5 & 0.5 & 0.552 & 0.007 \\
\hline 0.4897 & $\mathrm{R}$ & 304.4 & 0.3 & 0.489 & 0.006 \\
\hline 0.4897 & $\mathrm{R}$ & 114.2 & 0.2 & 0.879 & 0.008 \\
\hline 0.4897 & $\mathrm{R}$ & 358.1 & 0.4 & 0.822 & 0.008 \\
\hline 0.4898 & $\mathrm{R}$ & 159.4 & 0.6 & 0.457 & 0.006 \\
\hline 0.4898 & $\mathrm{R}$ & 132.3 & 0.4 & 0.548 & 0.007 \\
\hline 0.4898 & $\mathrm{R}$ & 343.8 & 0.4 & 0.972 & 0.009 \\
\hline 0.4898 & $\mathrm{R}$ & 150.5 & 0.2 & 0.455 & 0.006 \\
\hline 0.4898 & $\mathrm{R}$ & 53.7 & 0.9 & 0.731 & 0.008 \\
\hline 0.4898 & $\mathrm{R}$ & 252.4 & 0.2 & 0.186 & 0.004 \\
\hline 0.4898 & $\mathrm{R}$ & 214.4 & 0.3 & 0.273 & 0.005 \\
\hline 0.4953 & $\mathrm{R}$ & 125.4 & 0.3 & 0.667 & 0.007 \\
\hline 0.4953 & $\mathrm{R}$ & 121.4 & 0.3 & 1.097 & 0.010 \\
\hline 0.4953 & $\mathrm{R}$ & 166.4 & 0.4 & 0.209 & 0.004 \\
\hline 0.4953 & $\mathrm{R}$ & 284.5 & 0.3 & 0.454 & 0.006 \\
\hline 0.4953 & $\mathrm{R}$ & 173.4 & 0.2 & 0.621 & 0.007 \\
\hline 0.4953 & $\mathrm{R}$ & 91.7 & 0.3 & 0.788 & 0.008 \\
\hline 0.4953 & $\mathrm{R}$ & 256.2 & 0.3 & 1.436 & 0.012 \\
\hline 0.4953 & $\mathrm{R}$ & 286.4 & 0.2 & 0.149 & 0.004 \\
\hline 0.4953 & $\mathrm{R}$ & 135.4 & 0.3 & 0.340 & 0.005 \\
\hline 0.4953 & $\mathrm{R}$ & 37.3 & 0.3 & 0.788 & 0.008 \\
\hline 0.4953 & $\mathrm{R}$ & 52.9 & 0.2 & 0.914 & 0.009 \\
\hline 0.4953 & $\mathrm{R}$ & 216.4 & 0.3 & 0.184 & 0.004 \\
\hline 0.4953 & $\mathrm{R}$ & 205.4 & 0.2 & 0.333 & 0.005 \\
\hline 0.4953 & $\mathrm{R}$ & 213.4 & 0.6 & 0.211 & 0.005 \\
\hline 0.4953 & $\mathrm{R}$ & 245.4 & 0.2 & 0.242 & 0.005 \\
\hline 0.4953 & $\mathrm{R}$ & 187.4 & 0.2 & 0.641 & 0.007 \\
\hline 0.4953 & $\mathrm{R}$ & 81.4 & 0.3 & 0.762 & 0.008 \\
\hline 0.4953 & $\mathrm{R}$ & 19.5 & 1.6 & 1.676 & 0.014 \\
\hline 0.4953 & $\mathrm{R}$ & 8.6 & 0.3 & 0.427 & 0.006 \\
\hline 0.4953 & $\mathrm{R}$ & 178.4 & 0.4 & 0.214 & 0.005 \\
\hline 0.4953 & $\mathrm{R}$ & 125.5 & 0.2 & 0.880 & 0.008 \\
\hline 0.4898 & $\mathrm{R}$ & 161.6 & 0.5 & 0.642 & 0.007 \\
\hline 0.4953 & $\mathrm{R}$ & 338.4 & 0.3 & 0.976 & 0.009 \\
\hline 0.4898 & $\mathrm{R}$ & 6.4 & 0.2 & 0.851 & 0.008 \\
\hline 0.4898 & $\mathrm{R}$ & 290.4 & 0.3 & 0.822 & 0.008 \\
\hline 0.4898 & $\mathrm{R}$ & 305.4 & 0.6 & 0.214 & 0.005 \\
\hline 0.4898 & $\mathrm{R}$ & 156.4 & 0.5 & 0.244 & 0.005 \\
\hline 0.4899 & $\mathrm{R}$ & 299.4 & 0.2 & 0.520 & 0.006 \\
\hline 0.4899 & $\mathrm{R}$ & 76.1 & 0.4 & 0.761 & 0.008 \\
\hline 0.4899 & $\mathrm{R}$ & 247.4 & 0.3 & 0.697 & 0.007 \\
\hline 0.4899 & $\mathrm{R}$ & 161.4 & 0.5 & 0.393 & 0.006 \\
\hline
\end{tabular}


TABLE 2. (CONTINUED)

\begin{tabular}{|c|c|c|c|c|c|c|c|}
\hline $20349+1120$ & TDT2394 & 0.4899 & $\mathrm{R}$ & 1.5 & 0.2 & 0.455 & 0.006 \\
\hline $20351-0436$ & RST4671 & 0.4899 & $\mathrm{R}$ & 227.5 & 0.2 & 0.730 & 0.008 \\
\hline $20354+1121$ & YR 16 & 0.4899 & $\mathrm{R}$ & 278.4 & 0.4 & 0.701 & 0.008 \\
\hline $20381+2953$ & A 744 & 0.4925 & $\mathrm{R}$ & 274.3 & 0.2 & 0.732 & 0.008 \\
\hline $20384+2455$ & TDT2446 & 0.4925 & $\mathrm{R}$ & 99.5 & 0.4 & 0.459 & 0.006 \\
\hline $20385+2945$ & COU1172 & 0.4925 & $\mathrm{R}$ & 279.5 & 0.4 & 0.336 & 0.005 \\
\hline $20386+2007$ & COU 225 & 0.4925 & $\mathrm{R}$ & 286.4 & 0.8 & 0.277 & 0.005 \\
\hline $20390+3702$ & COU2219 & 0.4981 & $\mathrm{R}$ & 45.2 & 0.3 & 0.941 & 0.009 \\
\hline $20393+2714$ & TDT2454 Ba,Bb & 0.4926 & $\mathrm{R}$ & 46.3 & 0.3 & 0.916 & 0.009 \\
\hline $20397+3658$ & A 1432 & 0.4981 & $\mathrm{R}$ & 117.4 & 0.3 & 0.432 & 0.006 \\
\hline $20401+3044$ & TDT2457 Aa,Ab & 0.4926 & $\mathrm{R}$ & 138.5 & 0.4 & 0.888 & 0.009 \\
\hline $20406+2156$ & A 2795 & 0.4926 & $\mathrm{R}$ & 240.4 & 0.2 & 0.246 & 0.005 \\
\hline $20410+3218$ & STF2716 AB & 0.4981 & $\mathrm{R}$ & 46.4 & 0.1 & 2.772 & 0.020 \\
\hline $20411+2751 *$ & TDT2471 & 0.4926 & $\mathrm{R}$ & 2.4 & 0.8 & 0.240 & 0.005 \\
\hline $20411+3516$ & COU1963 AB,C & 0.4981 & $\mathrm{R}$ & 48.4 & 0.2 & 1.645 & 0.013 \\
\hline $20411+3516$ & COU1963 AB & 0.4981 & $\mathrm{R}$ & 180.4 & 0.2 & 0.213 & 0.004 \\
\hline $20416+3000$ & COU1174 & 0.4926 & $\mathrm{R}$ & 32.4 & 0.4 & 0.339 & 0.005 \\
\hline $20416+3950$ & COU2290 & 0.4981 & $\mathrm{R}$ & 43.3 & 0.2 & 0.545 & 0.006 \\
\hline $20424+3455$ & COU1965 & 0.4981 & $\mathrm{R}$ & 277.5 & 0.2 & 0.365 & 0.005 \\
\hline $20432+3350$ & HDS2949 & 0.4981 & $\mathrm{R}$ & 166.4 & 0.2 & 0.944 & 0.009 \\
\hline $20433+2616$ & COU1039 & 0.4926 & $\mathrm{R}$ & 237.1 & 0.4 & 1.035 & 0.009 \\
\hline $20440+3839$ & COU2292 & 0.4981 & $\mathrm{R}$ & 240.4 & 0.2 & 0.309 & 0.005 \\
\hline $20445+3409$ & HU 690 & 0.4981 & $\mathrm{R}$ & 281.5 & 0.2 & 0.490 & 0.006 \\
\hline $20447+2703 *$ & TDT2515 & 0.4926 & $\mathrm{R}$ & 277.5 & 0.6 & 0.400 & 0.006 \\
\hline $20451+3529$ & COU1809 & 0.4981 & $\mathrm{R}$ & 101.4 & 0.3 & 0.731 & 0.008 \\
\hline $20459+3852 *$ & COU2294 & 0.4982 & $\mathrm{R}$ & 124.9 & 0.3 & 0.857 & 0.008 \\
\hline $20460+3554 *$ & TDT2525 & 0.4982 & $\mathrm{R}$ & 70.3 & 0.3 & 0.579 & 0.007 \\
\hline $20463+2853 *$ & TDT2530 & 0.4926 & $\mathrm{R}$ & 207.3 & 0.3 & 0.606 & 0.007 \\
\hline $20464+3511$ & COU1810 & 0.4982 & $\mathrm{R}$ & 183.4 & 0.5 & 0.187 & 0.004 \\
\hline $20475+3016$ & COU1176 & 0.4926 & $\mathrm{R}$ & 225.4 & 0.4 & 0.366 & 0.005 \\
\hline $20477+3258$ & COU1634 & 0.4982 & $\mathrm{R}$ & 61.4 & 0.4 & 0.395 & 0.006 \\
\hline $20480+3917$ & A $1434 \mathrm{AB}, \mathrm{C}$ & 0.4982 & $\mathrm{R}$ & 255.9 & 0.2 & 2.513 & 0.018 \\
\hline $20490+2540 *$ & HDS2966 & 0.4926 & $\mathrm{R}$ & 229.4 & 0.2 & 0.518 & 0.006 \\
\hline $20490+2637$ & COU 828 AB & 0.4927 & $\mathrm{R}$ & 188.5 & 0.2 & 0.913 & 0.009 \\
\hline $20490+3619$ & COU1811 & 0.4982 & $\mathrm{R}$ & 255.4 & 0.3 & 0.759 & 0.008 \\
\hline $20503+5937$ & MLR 239 & 0.4954 & $\mathrm{R}$ & 286.3 & 0.3 & 0.888 & 0.009 \\
\hline $20531+2909$ & STT $417 \mathrm{AB}$ & 0.4927 & $\mathrm{R}$ & 28.5 & 0.2 & 0.917 & 0.009 \\
\hline $20535+2630$ & COU1177 & 0.4927 & $\mathrm{R}$ & 14.4 & 0.4 & 0.248 & 0.005 \\
\hline $20547+2516$ & COU 830 & 0.4927 & $\mathrm{R}$ & 168.8 & 0.3 & 1.039 & 0.009 \\
\hline $20573+2345 *$ & TDT2656 & 0.4927 & $\mathrm{R}$ & 349.4 & 0.2 & 0.735 & 0.008 \\
\hline $21009+5929$ & MLR 241 & 0.4954 & $\mathrm{R}$ & 174.6 & 0.2 & 0.948 & 0.009 \\
\hline $21012+5953 *$ & TDT2696 & 0.4954 & $\mathrm{R}$ & 67.3 & 0.3 & 0.519 & 0.006 \\
\hline $21035+5925$ & MLR 243 & 0.4954 & $\mathrm{R}$ & 224.4 & 0.6 & 0.211 & 0.005 \\
\hline $21036+5358$ & HDS2999 Aa,Ab & 0.4954 & $\mathrm{R}$ & 351.5 & 0.2 & 0.337 & 0.005 \\
\hline $21055+5340$ & $\mathrm{BU} 680 \mathrm{AB}$ & 0.4954 & $\mathrm{R}$ & 284.5 & 0.2 & 0.635 & 0.007 \\
\hline $21067+5556$ & TDT2753 & 0.4954 & $\mathrm{R}$ & 211.4 & 0.3 & 0.704 & 0.007 \\
\hline $21096+0550 *$ & TDT2793 & 0.4899 & $\mathrm{R}$ & 173.4 & 0.5 & 0.336 & 0.005 \\
\hline $21106+1650$ & HU 367 & 0.4899 & $\mathrm{R}$ & 339.4 & 0.2 & 0.331 & 0.005 \\
\hline $21118+5959$ & STF2780 AB & 0.4954 & $\mathrm{R}$ & 214.1 & 0.1 & 1.037 & 0.009 \\
\hline $21119+2758 *$ & TDT2814 & 0.4927 & $\mathrm{R}$ & 290.4 & 0.4 & 2.254 & 0.017 \\
\hline $21142+1231$ & HEI 406 & 0.4899 & $\mathrm{R}$ & 180.2 & 0.3 & 0.704 & 0.007 \\
\hline $21152+2753$ & COU 531 & 0.4927 & $\mathrm{R}$ & 144.2 & 0.2 & 0.943 & 0.009 \\
\hline $21152+5531$ & A 1692 & 0.4954 & $\mathrm{R}$ & 167.4 & 0.2 & 0.272 & 0.005 \\
\hline $21160+5914 *$ & TDT2869 & 0.4954 & $\mathrm{R}$ & 113.3 & 0.5 & 0.674 & 0.007 \\
\hline $21196+5552$ & MLR 582 & 0.4954 & $\mathrm{R}$ & 347.5 & 0.3 & 0.948 & 0.009 \\
\hline $21197+5455$ & A 1694 & 0.4954 & $\mathrm{R}$ & 94.6 & 0.2 & 0.850 & 0.008 \\
\hline $21199+5319$ & A 1695 & 0.4954 & $\mathrm{R}$ & 193.5 & 0.2 & 0.483 & 0.006 \\
\hline $21200+5436$ & TDT2908 Aa,Ab & 0.4954 & $\mathrm{R}$ & 115.4 & 0.5 & 0.306 & 0.005 \\
\hline $21202+5411$ & HDS3036 & 0.4954 & $\mathrm{R}$ & 165.4 & 0.2 & 0.392 & 0.005 \\
\hline $21203+5354$ & TDT2910 & 0.4954 & $\mathrm{R}$ & 286.6 & 0.2 & 0.675 & 0.007 \\
\hline $21227+5214$ & HU 591 & 0.4954 & $\mathrm{R}$ & 129.5 & 0.2 & 0.761 & 0.008 \\
\hline
\end{tabular}


TABLE 2. (CONTINUED)

\begin{tabular}{|c|c|c|c|c|c|c|c|}
\hline $21237+5518$ & A 1892 & 0.4954 & $\mathrm{R}$ & 350.1 & 0.2 & 0.766 & 0.008 \\
\hline $21249+5734$ & A 766 & 0.4954 & $\mathrm{R}$ & 228.3 & 0.3 & 0.486 & 0.006 \\
\hline $21251+5229$ & HU 592 & 0.4954 & $\mathrm{R}$ & 325.4 & 0.2 & 0.823 & 0.008 \\
\hline $21252+5618$ & TDS1127 & 0.4954 & $\mathrm{R}$ & 235.7 & 0.2 & 1.097 & 0.010 \\
\hline $21273+5953$ & MLR 362 & 0.4954 & $\mathrm{R}$ & 55.5 & 0.3 & 0.338 & 0.005 \\
\hline $21274+5835$ & MLR 435 & 0.4954 & $\mathrm{R}$ & 236.4 & 0.5 & 0.181 & 0.004 \\
\hline $21287+5710$ & BU 1142 & 0.4954 & $\mathrm{R}$ & 5.5 & 0.4 & 0.369 & 0.005 \\
\hline $21327+5459 *$ & HDS3063 & 0.4954 & $\mathrm{R}$ & 2.4 & 0.2 & 0.339 & 0.005 \\
\hline $21346+5633$ & A $1893 \mathrm{AB}$ & 0.4954 & $\mathrm{R}$ & 28.3 & 0.2 & 0.641 & 0.007 \\
\hline $21362+5139$ & HDS3075 & 0.4954 & $\mathrm{R}$ & 15.9 & 0.2 & 0.609 & 0.007 \\
\hline $21372+5346$ & MLR 609 & 0.4954 & $\mathrm{R}$ & 40.5 & 0.2 & 1.035 & 0.009 \\
\hline $21376+5546$ & $\mathrm{BU} 686 \mathrm{AB}$ & 0.4954 & $\mathrm{R}$ & 311.2 & 0.2 & 1.004 & 0.009 \\
\hline $21377+5659$ & MLR 583 & 0.4954 & $\mathrm{R}$ & 15.2 & 0.3 & 0.789 & 0.008 \\
\hline $21377+5734$ & D $25 \mathrm{AB}$ & 0.4955 & $\mathrm{R}$ & 163.6 & 0.2 & 0.974 & 0.009 \\
\hline $21378+5333 *$ & TDT3067 & 0.4955 & $\mathrm{R}$ & 33.6 & 0.2 & 0.821 & 0.008 \\
\hline $21398+5403$ & TDT3093 & 0.4955 & $\mathrm{R}$ & 332.5 & 0.4 & 0.398 & 0.006 \\
\hline $22016+5654 *$ & TDT3295 & 0.4955 & $\mathrm{R}$ & 42.4 & 1.1 & 0.179 & 0.005 \\
\hline $22033+5403 *$ & TDT3313 & 0.4955 & $\mathrm{R}$ & 133.3 & 0.4 & 0.886 & 0.009 \\
\hline $22045+5239$ & HU 776 & 0.4955 & $\mathrm{R}$ & 357.4 & 0.8 & 0.164 & 0.004 \\
\hline $22056+5711$ & $\mathrm{BAR} 57 \mathrm{AB}$ & 0.4955 & $\mathrm{R}$ & 274.5 & 0.4 & 0.976 & 0.009 \\
\hline $22075+5631$ & HDS3141 & 0.4955 & $\mathrm{R}$ & 330.5 & 0.2 & 0.514 & 0.006 \\
\hline $22077+5020$ & COU2550 & 0.4955 & $\mathrm{R}$ & 114.2 & 0.2 & 0.673 & 0.007 \\
\hline $22078+5333$ & MLR 592 & 0.4955 & $\mathrm{R}$ & 22.4 & 0.3 & 0.488 & 0.006 \\
\hline $22080+5635$ & HDS3144 & 0.4955 & $\mathrm{R}$ & 33.4 & 0.2 & 0.166 & 0.004 \\
\hline $22093+5804$ & MLR 557 & 0.4955 & $\mathrm{R}$ & 304.6 & 0.3 & 0.970 & 0.009 \\
\hline $22107+5830$ & A 624 & 0.4955 & $\mathrm{R}$ & 14.4 & 0.2 & 0.763 & 0.008 \\
\hline $22115+5110$ & COU2660 & 0.4955 & $\mathrm{R}$ & 250.2 & 0.3 & 0.670 & 0.007 \\
\hline $22115+5232$ & COU2659 & 0.4955 & $\mathrm{R}$ & 161.9 & 0.3 & 1.253 & 0.011 \\
\hline $22117+5743$ & A $625 \mathrm{AB}$ & 0.4955 & $\mathrm{R}$ & 81.5 & 0.2 & 0.551 & 0.006 \\
\hline $22122+5909$ & MLR 439 & 0.4955 & $\mathrm{R}$ & 253.6 & 0.2 & 0.791 & 0.008 \\
\hline
\end{tabular}

TABLE 3

ASTROMETRIC MEASUREMENTS AND RESIDUALS FOR OBSERVED BINARY STARS WITH CALCULATED ORBITS

\begin{tabular}{|c|c|c|c|c|c|c|c|c|c|c|}
\hline $\begin{array}{l}\text { WDS } \\
(2000)\end{array}$ & $\begin{array}{c}\text { Discoverer } \\
\text { designation }\end{array}$ & $\begin{array}{l}\text { Epoch } \\
2016+\end{array}$ & Fil. & $\begin{array}{c}\theta \\
\left({ }^{\circ}\right)\end{array}$ & $\begin{array}{l}\delta \theta \\
\left({ }^{\circ}\right)\end{array}$ & $\begin{array}{c}\rho \\
\left({ }^{\prime \prime}\right)\end{array}$ & $\begin{array}{l}\delta \rho \\
\left({ }^{\prime \prime}\right)\end{array}$ & $\begin{array}{c}\theta_{O}-\theta_{C} \\
\left({ }^{\circ}\right)\end{array}$ & $\begin{array}{c}\rho_{O}-\rho_{C} \\
\left({ }^{\prime \prime}\right)\end{array}$ & $\begin{array}{c}\text { Orbit } \\
\text { Ref. }\end{array}$ \\
\hline $14190-0636$ & HDS2016 AB & 0.4919 & $\mathrm{R}$ & 325.9 & 0.3 & 0.198 & 0.004 & 1.5 & 0.011 & Tok2015c \\
\hline $14231+0729$ & A 1104 & 0.4919 & $\mathrm{R}$ & 244.4 & 0.2 & 0.423 & 0.006 & 1.3 & -0.058 & Izm2019 \\
\hline $14267+1625$ & A 2069 & 0.4919 & $\mathrm{R}$ & 102.4 & 0.3 & 0.159 & 0.004 & 24.8 & 0.034 & Sca2001g \\
\hline $14426+1929$ & $\mathrm{HU} 575 \mathrm{AB}$ & 0.4920 & $\mathrm{R}$ & 115.4 & 0.2 & 0.368 & 0.005 & 0.5 & 0.003 & Sod1999 \\
\hline $16038+1406$ & HDS2265 & 0.4921 & $\mathrm{R}$ & 9.4 & 0.3 & 0.276 & 0.005 & 0.1 & 0.000 & Tok2018e \\
\hline $16059+1041$ & $\mathrm{HDS} 2273 \mathrm{Aa}, \mathrm{Ab}$ & 0.4921 & $\mathrm{R}$ & 253.5 & 0.2 & 0.364 & 0.005 & 0.4 & 0.009 & Tok2019h \\
\hline $16115+1507$ & A 1799 & 0.4921 & $\mathrm{R}$ & 296.6 & 0.2 & 0.817 & 0.008 & 1.4 & 0.024 & Zir2014a \\
\hline $16169+0113$ & A 2181 & 0.4921 & $\mathrm{R}$ & 86.5 & 0.3 & 0.518 & 0.006 & -18.8 & 0.101 & Pop1995d \\
\hline $17066+0039$ & TOK $52 \mathrm{Ba}, \mathrm{Bb}$ & 0.4922 & $\mathrm{R}$ & 18.4 & 0.2 & 0.091 & 0.004 & -4.8 & -0.008 & Izm2019 \\
\hline $17066+0039$ & $\mathrm{BU} 823 \mathrm{AB}$ & 0.4922 & $\mathrm{R}$ & 172.0 & 0.2 & 1.037 & 0.009 & 0.7 & -0.004 & Izm2019 \\
\hline $17136+1716$ & A 2087 & 0.4923 & $\mathrm{R}$ & 133.4 & 0.3 & 0.488 & 0.006 & -1.8 & 0.016 & Mnt2001a \\
\hline $17155+1052$ & HDS2440 & 0.4923 & $\mathrm{R}$ & 113.4 & 0.5 & 0.160 & 0.004 & -7.5 & 0.021 & Cve2014 \\
\hline $17176+1025$ & HDS2445 & 0.4923 & $\mathrm{R}$ & 261.4 & 0.3 & 0.215 & 0.004 & 1.5 & -0.002 & Tok2017b \\
\hline $17240+3835$ & HU 1179 & 0.4977 & $\mathrm{R}$ & 272.4 & 0.1 & 0.303 & 0.005 & 3.1 & 0.039 & Hrt2000b \\
\hline $17251+3444$ & $\mathrm{HU} 922 \mathrm{Aa}, \mathrm{Ab}$ & 0.4977 & $\mathrm{R}$ & 30.4 & 0.3 & 0.275 & 0.005 & 0.4 & -0.009 & FMR2016b \\
\hline $17487+3536$ & HU 1182 & 0.4977 & $\mathrm{R}$ & 298.5 & 0.2 & 0.426 & 0.006 & -0.8 & 0.021 & USN2002 \\
\hline $17490+3704$ & COU1145 & 0.4977 & $\mathrm{R}$ & 103.4 & 0.2 & 0.164 & 0.004 & 1.0 & 0.017 & Hrt1996a \\
\hline $17591+3228$ & HU 1185 & 0.4977 & $\mathrm{R}$ & 145.5 & 0.2 & 0.394 & 0.006 & 1.2 & -0.005 & Doc2012i \\
\hline $18003+2154$ & A $1374 \mathrm{AB}$ & 0.4950 & $\mathrm{R}$ & 215.4 & 0.2 & 0.485 & 0.006 & 2.2 & -0.012 & Msn2017a \\
\hline $18017+4011$ & STF2267 & 0.4950 & $\mathrm{R}$ & 277.5 & 0.2 & 0.521 & 0.006 & 1.9 & 0.000 & Zir2014a \\
\hline $18025+4414$ & $\mathrm{BU} 1127 \mathrm{AB}$ & 0.4950 & $\mathrm{R}$ & 46.3 & 0.2 & 0.701 & 0.007 & 0.7 & 0.000 & Cve2016c \\
\hline $18033+3921$ & STF2275 & 0.4977 & $\mathrm{R}$ & 303.5 & 0.2 & 0.336 & 0.005 & -1.5 & 0.023 & Pop2000a \\
\hline
\end{tabular}


TABLE 3. (CONTINUED)

\begin{tabular}{|c|c|c|c|c|c|c|c|c|c|c|}
\hline $18033+3921$ & STF2275 & 0.4950 & $\mathrm{R}$ & 303.5 & 0.2 & 0.335 & 0.005 & -1.4 & 0.022 & Pop2000a \\
\hline $18035+4032$ & COU1785 & 0.4950 & $\mathrm{R}$ & 30.4 & 0.2 & 0.166 & 0.004 & -5.0 & 0.000 & Doc2008a \\
\hline $18043+4206$ & COU1786 Aa,Ab & 0.4950 & $\mathrm{R}$ & 11.4 & 0.2 & 0.152 & 0.004 & 12.0 & -0.030 & Hrt2009 \\
\hline $18063+3824$ & HU 1186 & 0.4978 & $\mathrm{R}$ & 134.4 & 0.2 & 0.176 & 0.004 & -29.7 & 0.104 & USN2006b \\
\hline $18092+3129$ & COU 812 & 0.4923 & $\mathrm{R}$ & 265.6 & 0.3 & 0.672 & 0.007 & -21.0 & -0.006 & Cou1999b \\
\hline $18097+5024$ & HU 674 & 0.4951 & $\mathrm{R}$ & 214.4 & 0.2 & 0.765 & 0.008 & 0.6 & 0.024 & Msn2017e \\
\hline $18126+3836$ & BU 1091 & 0.4978 & $\mathrm{R}$ & 320.3 & 0.2 & 0.765 & 0.008 & 1.5 & 0.004 & Zir2012b \\
\hline $18130+3318$ & COU1006 & 0.4978 & $\mathrm{R}$ & 337.4 & 0.2 & 0.514 & 0.006 & 81.6 & 0.220 & Cou1999b \\
\hline $18163+3625$ & HU 1291 & 0.4978 & $\mathrm{R}$ & 52.4 & 0.2 & 0.304 & 0.005 & 3.0 & 0.017 & Hrt2014b \\
\hline $18250-0135$ & $\mathrm{AC} 11$ & 0.4895 & $\mathrm{R}$ & 355.7 & 0.1 & 0.914 & 0.009 & 0.8 & 0.005 & Tok2017c \\
\hline $18261+0047$ & BU 1203 & 0.4896 & $\mathrm{R}$ & 158.3 & 0.2 & 0.518 & 0.006 & 0.4 & 0.021 & Pop1996b \\
\hline $18320+0647$ & STT 354 & 0.4897 & $\mathrm{R}$ & 217.3 & 0.2 & 0.549 & 0.006 & 2.5 & -0.026 & Zir2013a \\
\hline $18339+5221$ & A $1377 \mathrm{AB}$ & 0.4951 & $\mathrm{~V}$ & 134.4 & 0.1 & 0.244 & 0.005 & -6.3 & 0.109 & Mut2010e \\
\hline $18421+3445$ & B $2546 \mathrm{Aa}, \mathrm{Ab}$ & 0.4978 & $\mathrm{R}$ & 24.4 & 0.2 & 0.124 & 0.004 & -2.5 & 0.018 & USN2002 \\
\hline $18437+3141$ & A 253 & 0.4979 & $\mathrm{R}$ & 134.2 & 0.2 & 0.702 & 0.007 & -2.2 & 0.094 & Baz1987d \\
\hline $18466+3821$ & HU 1191 & 0.4979 & $\mathrm{R}$ & 338.4 & 0.2 & 0.218 & 0.004 & -2.2 & -0.009 & Doc $2009 \mathrm{~g}$ \\
\hline $19039+2642$ & A 2992 & 0.4925 & $\mathrm{R}$ & 224.4 & 0.4 & 0.167 & 0.004 & -3.7 & -0.018 & Doc $2009 \mathrm{~g}$ \\
\hline $19055+3352$ & HU 940 & 0.4979 & $\mathrm{R}$ & 191.4 & 0.2 & 0.458 & 0.006 & 2.0 & -0.004 & Doc2009g \\
\hline $19073+2432$ & A 262 & 0.4925 & $\mathrm{R}$ & 269.4 & 0.3 & 0.167 & 0.004 & -3.2 & -0.034 & Zir2012b \\
\hline $19083+2706$ & HO $98 \mathrm{AB}$ & 0.4925 & $\mathrm{R}$ & 61.4 & 0.2 & 0.209 & 0.004 & 5.3 & 0.030 & Lin2012a \\
\hline $19083+5520$ & D $19 \mathrm{AB}$ & 0.4952 & $\mathrm{R}$ & 344.5 & 0.2 & 0.458 & 0.006 & 1.6 & -0.034 & Hrt2013c \\
\hline $19106+5429$ & A 1391 & 0.4952 & $\mathrm{R}$ & 22.4 & 0.3 & 0.246 & 0.005 & -2.7 & 0.013 & Pru2014 \\
\hline $19216+5223$ & BU 1129 & 0.4952 & $\mathrm{R}$ & 343.4 & 0.2 & 0.304 & 0.005 & 2.9 & -0.014 & Baz1984a \\
\hline $19296+1224$ & A 1653 & 0.4897 & $\mathrm{R}$ & 142.4 & 0.2 & 0.210 & 0.004 & 7.1 & 0.013 & Pru2014 \\
\hline $19330+0546$ & A 367 & 0.4898 & $\mathrm{R}$ & 306.3 & 0.2 & 1.037 & 0.009 & 2.2 & 0.078 & Izm2019 \\
\hline $19351+5038$ & HU 679 & 0.4953 & $\mathrm{R}$ & 272.5 & 0.2 & 0.397 & 0.005 & 3.3 & 0.001 & Ana2005 \\
\hline $20306+1349$ & HDS2932 & 0.4898 & $\mathrm{R}$ & 309.4 & 0.4 & 0.122 & 0.004 & -10.1 & -0.017 & Hor 2011b \\
\hline $20311+1548$ & A 1675 & 0.4898 & $\mathrm{R}$ & 304.4 & 0.2 & 0.165 & 0.004 & 0.8 & 0.004 & Hrt2001b \\
\hline $20312+1116$ & CHR $99 \mathrm{Aa}, \mathrm{Ab}$ & 0.4898 & $\mathrm{R}$ & 185.3 & 0.2 & 0.307 & 0.005 & -2.8 & -0.030 & Hrt2014b \\
\hline $20329+1357$ & L 35 CD & 0.4898 & $\mathrm{R}$ & 143.5 & 0.3 & 0.515 & 0.006 & -0.1 & 0.032 & Hrt2014b \\
\hline $20410+3905$ & $\mathrm{MCA} 62 \mathrm{Aa}, \mathrm{Ab}$ & 0.4981 & $\mathrm{R}$ & 276.4 & 0.2 & 0.122 & 0.004 & -1.6 & 0.034 & Ole2003c \\
\hline $20444+1945$ & CAR 2 & 0.4926 & $\mathrm{R}$ & 320.4 & 0.8 & 0.218 & 0.005 & 13.1 & 0.008 & Cve2017b \\
\hline $21109+2925$ & BAG 29 & 0.4927 & $\mathrm{R}$ & 174.4 & 0.7 & 0.188 & 0.004 & 1.2 & 0.000 & Bag2010 \\
\hline $21125+2821$ & HO 152 & 0.4927 & $\mathrm{R}$ & 158.4 & 0.3 & 0.167 & 0.004 & 6.2 & 0.026 & Doc $2016 \mathrm{~g}$ \\
\hline $21135+0713$ & $\mathrm{BU} 270 \mathrm{AB}$ & 0.4899 & $\mathrm{R}$ & 345.4 & 0.2 & 0.490 & 0.006 & 0.7 & 0.008 & Msn2017a \\
\hline $21147-0050$ & A 883 AB & 0.4899 & $\mathrm{R}$ & 287.4 & 0.3 & 0.160 & 0.004 & 0.5 & 0.013 & Hrt2009 \\
\hline $22086+5917$ & STF2872 BC & 0.4955 & $\mathrm{R}$ & 298.2 & 0.2 & 0.848 & 0.008 & 1.3 & 0.046 & USN2002 \\
\hline
\end{tabular}

TABLE 4

NEW CLOSE DOUBLE STARS

\begin{tabular}{ccccrr}
\hline Identifier & $\begin{array}{c}\text { Coordinates } \\
\text { RA \& DEC (2000) }\end{array}$ & $\begin{array}{c}\text { Flux } \\
m_{V}\end{array}$ & $\begin{array}{c}\text { Epoch } \\
2016+\end{array}$ & $\begin{array}{c}\text { Fil. } \\
\left(\begin{array}{c}\rho \\
\left({ }^{\prime}\right)\end{array}\right.\end{array}$ & $\begin{array}{c}\theta \\
\left({ }^{\circ}\right)\end{array}$ \\
\hline TYC 416-564-1 & $174554.92+013456.60$ & 10.96 & 0.4893 & I & $349.4 \pm 0.3$ \\
TYC 420-1003-1 & $174616.60+015645.87$ & 11.35 & 0.4893 & I & $265.4 \pm 0.4$ \\
TYC 416-174-1 & $174621.64+012208.59$ & 10.07 & 0.4893 & I & $11.6 \pm 0.2$ \\
\hline
\end{tabular}

format used in the WDS Catalog (Worley \& Douglass 1997). The second column gives the official binary star discoverer designation. The third column gives the epoch of the observation in fractional Julian years. The fourth column indicates the filter used. The two following columns contain the measured position angles given in degrees, with the errors of their determination, and the angular separation in arcseconds, with the errors of its determination.
The astrometric measurements of close double stars without known orbits are displayed in Table 2. The symbol $(*)$ indicates that this system was previously discovered but never confirmed. We confirm these systems as double stars. However, for many of them, the current position of the component is far away from the one reported previously. Therefore, it is uncertain to determine whether it is a confirmation or a new pair. The second column gives the 
official binary star discoverer designation. The last four columns give the position angle $\theta$ (Column 5) with its error $\sigma \theta$ (Column 6) in degrees, and the angular separation $\rho$ (Column 7) with its error $\sigma \rho$ (Column 8) in arcseconds.

Furthermore, we have observed 65 close binary stars with known orbits from the Sixth Catalog of Orbits of Visual Binary Stars (OC6) (Hartkopf et al. 2001). The astrometric measurements are displayed in Table 3. The first 8 columns are the same as in Table 2. The last three columns give the difference between our measurements and the ephemeris calculated for the date of observation, as well as references in the format of OC6. The orbital elements and the complete list of references may be found in the current electronic version of OC6: http: //ad.usno.navy.mil/wds/orb6.html.

The last Table 4 displays the astrometric parameters of three new close double stars with separation less than one arcsecond.

The astrometric results include errors arising in the process of recovering the component positions from the power spectrum. In addition, the position angle measure $(\theta)$ can have a systematic error of $0.14^{\circ}$ and the separation measure $(\rho)$ has an additional error pertaining to the pixel scale.

\section{CONCLUSIONS}

We present results of double star speckle interferometric observations focused on close binaries from the WDS catalog. We present the astrometric results for 468 resolved stars. We confirm 59 stars as doubles.

For astrometric measurements, we calculate the high resolution autocorrelation function in polar coordinates. It allows one to perform astrometric measurements even for a distorted power spectrum. The coordinates of the global maximum of $A C F_{p}$ corresponds to the $\rho$ and $\theta$ of the component. The measurements can be carried out without a speckle interferometric transfer function correction, because we exclude atmospheric distortion by using the window $W(r, \phi)$. Finally, the self-calibrating shift-and-add technique solves the 180 degree ambiguity.
This research is supported by the Dirección General de Asuntos del Personal Académico (UNAM, México) under project IN107818. Based upon observations acquired at the Observatorio Astronómico Nacional in the Sierra San Pedro Mártir (OAN-SPM), Baja California, México. We thank the daytime and night support staff at the OAN-SPM for facilitating and helping us to obtain our observations. We have made an extensive use of the SIMBAD and ADS services, for which we are thankful. Also, we would like to thank the reviewers for the time they spent on our manuscript and for their comments which helped us to improve it.

\section{REFERENCES}

Christou, J. C., Hege, E. K., Freeman, J. D., \& Ribak, E. 1986, JOSAA, 3, 204

Guerrero, C. A., Rosales-Ortega, F. F., Escobedo, G., et al. 2020, MNRAS, 495, 806

Hartkopf, W. I., Mason, B. D., \& Worley, C. E. 2001, AJ, 122,3472

Hartkopf, W. I., McAlister, H. A., \& Mason, B. D. 2001, AJ, 122, 3480

Kerp, J., Barth, W., Hofmann, K., Reinheimer, T., \& Weigelt, G. 1992, ESO Conference on HighResolution Imaging by Interferometry II. Part 1: ground interferometry and infrared wavelengths, ed. J. M. Beckers \& F. Merkle , 1, 269

Labeyrie, A. 1970, A\&A, 6, 85

Mitrofanova, A., Dyachenko, V., Beskakotov, A., et al. 2020, AJ, 159, 266

Orlov, V. G., Voitsekhovich, V. V., Mendoza-Valencia, G. A., et al. 2009, RMxAA, 45, 155

Tokovinin, A., Mason, B. D., \& Hartkopf, W. I. 2010, AJ, 139, 743

Tokovinin, A., Mason, B. D., Hartkopf, W. I., Mendez, R. A., \& Horch, E. P. 2015, AJ, 150, 50

Tokovinin, A., Mason, B. D., Mendez, R. A., Costa, E., \& Horch, E. P. 2020, AJ, 160, 7

Worley, C. E. \& Douglass, G. G. 1997, A\&AS, 125, 523

V. G. Orlov: Instituto de Astronomía, Universidad Nacional Autónoma de México, Apdo. Postal 70-264, Cd. Universitaria, 04510 Ciudad de México, México (orlov@astro.unam.mx). 